\title{
EVALUATION OF A GRACE-BASED COMBINED GEOPOTENTIAL MODEL OVER THE BALTIC COUNTRIES
}

\author{
Artu Ellmann ${ }^{1}$, Harli Jürgenson ${ }^{2}$ \\ ${ }^{1}$ Tallinn University of Technology, Ehitajate str. 5, 19086 Tallinn, Estonia \\ E-mail: artu.ellmann@ttu.ee \\ ${ }^{2}$ Estonian University of Life Sciences, Kreutzwaldi 5, Tartu, Estonia \\ E-mail: harli.jyrgenson@emu.ee
}

Received 13-09-2007; accepted 12-03-2008

\begin{abstract}
Nowadays, many geodetic and engineering applications require that the two essential components of the vertical positioning - the height and the corresponding reference surface (geoid), are determined precisely. The recent advancements of satellite technology have resolved the long-wavelength component of the global geoid with an accuracy of a few $\mathrm{cm}$. The tracking data of the GRACE twin-satellites are the basis for the new combined geopotential model EIGEN-GL04c. This contribution assesses the quality of this model through comparisons with an earlier geopotential model (EGM96) of the Baltic countries. The method of spherical harmonic expansions is used in numerical investigations. The results of evaluation revealed significant discrepancies between the long wavelength contributions of the models, which may reach several decimetres in terms of the geoidal heights. There are also some notable improvements of numerical statistics (assessed by the GPS-levelling data) in the target area when utilizing the new EIGEN-GL04c, instead of using the EGM96. A decimetre level accuracy can be obtained for the EIGEN-GL04c derived geoid model, provided that any datum inconsistencies have been eliminated. This creates necessary preconditions to further improve the geoid models on the regional scale.
\end{abstract}

Keywords: geopotential model, Stokes's coefficients, GRACE, geoid, GPS-levelling, vertical datum, Baltic countries.

\section{Introduction}

The geoid is defined as an equipotential surface of the Earth's gravity field, (generally) inside the topographical masses on land and more or less coinciding with mean sea level at sea. The geoid is one of the most important definitions in the geosciences. It also plays an essential role in the national geodetic infrastructure, as the topographic heights and the depths of water bodies are determined by it. Due to irregularities in mass distributions inside the Earth, the geoidal heights undulate with respect to the reference ellipsoid. However, the deviations of the two surfaces do not exceed \pm 100 m globally. Specifically, the advance of the Global Positioning System (GPS) technology has increased the demand for a precise geoid model. Recall that at discrete points a conventional (sea level related) height (usually denoted by $H$ ) could be obtained by algebraically subtracting the value of the geoidal height from the geodetic height $(h$, acquired from the GPS observations).

According to Stokes (1849), the geoidal heights can be determined from the global distribution of the gravity anomalies. Yet, the application of the Stokes integral formula remains impractical, due to incomplete geographical coverage of the (terrestrial) gravity data. Broad characteristics of the global gravity field can be defined from the accurate tracking of LEO (Low Earth Orbit) satellites. In particular, the GRACE (Gravity Recovery and Climate Experiment) gravimetric mission has significantly improved the accuracy and spatial resolution of the gravity field parameters in the last few years. This improvement is mainly due to observations of the along-track perturbations of the GRACE twin-satellites. The satellite tracking data (along with other global data sources) are being used by authorized research centres to develop models of the Earth's gravity field. Such Global Geopotential Models (GGM) comprise a set of sphericalharmonic $(\mathrm{SH})$ coefficients, which are obtained from the spectral analysis of the geopotential. In other words, the gravity field is described as a wavelike surface built up by superimposing on each other a great number of waves of different wavelengths. Fourier transform is applied to convert the SH coefficients into a variety of gravimetric related quantities, such as the geoidal heights, gravity anomaly/disturbance and vertical deflections of gravity. Importantly, their spatial resolution is directly linked to the expansion degree of the Fourier series (the higher the degree, the better the spatial resolution). 
The satellite tracking data have resolved the longwavelength component of the global geoid with an accuracy of a few $\mathrm{cm}$. However, the spatial resolution of such information is limited to about $200 \mathrm{~km}$ (corresponding to the series expansion degree 100). Improvements to the Earth gravity models at medium and short wavelengths should come from the use of terrestrial surveys and satellite altimetry (over the oceans). This allows to enhance the spatial resolution of the combined models (in 2007 the best model resolution is $55 \mathrm{~km}$, corresponding to the expansion degree of 360).

The combined geopotential models have proved their value in many applications. A useful application of a GGM is to provide a basis for regional geoid modelling. Accordingly, the regional geoid improvements can be obtained by modifying the original Stokes integral formula. Modified Stokes's formula (Molodenskii et al. 1960) combines local terrestrial gravity anomalies and the GGM-derived long-wavelength component (i.e. the "global trend") of the geoid. Obviously, in this scenario the quality of the regional geoid models is highly dependent on the quality of the underlying GGM.

The tracking data of the GRACE twin-satellites are on the basis of a new combined geopotential model EIGEN-GL04c (Förste et al. 2006), which was recently released by the Potsdam GeoForschungs Zentrum (GFZ). Due to the data availability, quality, and type, the characteristics of a GGM vary regionally. Hence, the performance of any GGM needs to be validated on a regional scale. The focus of this study is on the Baltic countries Estonia, Latvia and Lithuania. The EIGEN-GL04c derived geoidal heights are compared with an earlier geopotential model, EGM96 (Lemoine et al. 1998), and with some ground "truth". This choice is due to the fact that the EGM96 has been employed in many regional and national geoid modellings over the Baltic countries. The EIGEN-GL04c/EGM96 discrepancies may also hint to possible deficiencies in the existing regional geoid solutions. The accuracy of the geoid models can be assessed by comparisons with other external data sets that depend on the same gravity field. GPS-levelling points are being employed for this task elsewhere and in the present study. The set-up of the relevant numerical investigations and the achieved results will be discussed in detail. A brief summary concludes the paper.

\section{Spectral decomposition of the gravity field, scaling of the zonal coefficents}

The gravitational potential and its derivatives (such as the disturbing potential, gravity anomaly and geoidal heights) can be expressed in terms of an infinite series of spherical harmonics outside the attracting masses of the Earth. At this, also the differences between the defining constants (i.e. gravity-mass constant; and the equatorial radius of the geodetic ellipsoid versus that of the GGM) of the used GGM and adopted geodetic reference ellipsoid need to be considered. Therefore a closer look to the scaling issues is in order.

The scaling can be introduced via zonal harmonics of the reference ellipsoid by an approach described in (Vaníček, Kleusberg 1987: Section 5). In the discussion below (see also Kirby, Featherstone 1997) the GGMrelated values will be denoted by the sub-/superscript "GGM", whereas the sub-/superscript "GRS" denotes the quantities associated with geodetic reference ellipsoid. Consider the gravitational potential $W_{G G M}$, which can be computed as

$$
\begin{aligned}
& W_{G G M}(r, \Omega)=\frac{G M_{G G M}}{r}\left(1+\sum_{n=2}^{n_{\max }}\left(\frac{a_{G G M}}{r}\right)^{n} \times\right. \\
& \sum_{m=0}^{n}\left\{\bar{C}_{n m}^{G G M} \cos m \lambda+\bar{S}_{n m}^{G G M} \cos m \lambda\right\} \bar{P}_{n m}(\cos \theta),
\end{aligned}
$$

where $r$ is the geocentric radius of the computation point, $\Omega$ denotes a pair of geocentric coordinates $(\theta, \lambda$; the spherical co-latitude and longitude, respectively), $\bar{C}_{n m}$ and $\bar{S}_{n m}$ are fully normalised spherical harmonic (Stokes') coefficients, of degree $n$ and order $m ; \bar{P}_{n m}(\cos \theta)$ are fully normalised associated Legendre functions, and GM is an adopted gravity mass constant. It should be noted that some recent geopotential models utilise $G M=$ $398600.4415 \mathrm{~km}^{3} \mathrm{~s}^{-2}$. Since the coefficients $\bar{C}_{n m}$ and $\bar{S}_{n m}$ are referred to the bounding sphere (with some radius $a$, the value $a=6378136.3 \mathrm{~m}$ is often adopted at the compilation of contemporary models), then the GGM-derived quantities, strictly speaking, ought be computed on the surface of the bounding sphere (or above it). However, the gravity field related quantities can be more or less safely computed inside this sphere, as long as the evaluation point remains outside the topographic masses. Due this, the GGM-s are better suited for computing the ground-related gravity quantities, such as the quasigeoid (a.k.a. height anomalies, cf., Molodenskii et al. 1960), rather than the geoid. Note that over the continents the latter would require computations inside the topographic masses, which violates the harmony condition. Importantly, the spatial wavelength and resolution of the GGM-derived gravity field parameters are directly linked to the maximum degree $n_{\max }$ of the series expansion. By convention, the wavelength is approximately the planet's circumference divided by the expansion degree, i.e., $\lambda=2 \pi a / n_{\max }\left(\lambda \approx 40000 / n_{\max }\right.$, in $\left.\mathrm{km}\right)$, whereas the spatial resolution is $\lambda / 2$.

The gravitational potential of the normal ellipsoid, i.e. the normal potential $U_{G R S}$, can be expressed via the zonal harmonic series

$$
\begin{aligned}
& U_{G R S}(r, \Omega)=\frac{G M_{G R S}}{r} \times \\
& \left(1+\sum_{n=2}^{10}\left(\frac{a_{G R S}}{r}\right)^{n} \bar{C}_{n 0}^{G R S} \bar{P}_{n 0}(\cos \theta)\right)
\end{aligned}
$$

where $a_{G R S}$ and $G M_{G R S}$ are the equatorial radius and the gravity-mass constant of the chosen reference ellipsoid, respectively. Note also the series in Eqs and (2) exclude the zero and first degree terms. As is customary in geodesy, the mass of the reference ellipsoid is chosen to be equal to the mass of the Earth, and the origin of the reference ellipsoid is placed at Earth's gravity centre. In this way the zero- and first-degree harmonics vanish. 
However, in reality the $G M_{G G M}$ and $a_{G G M}$ values may differ from the corresponding parameters of the adopted geodetic reference ellipsoid.

The disturbing potential $T_{G G M}$ is obtained by subtracting the normal potential (cf. Eq. (2)) from the actual gravitational potential (cf. Eq. ), i.e.,

$$
\begin{aligned}
& T_{G G M}(r, \Omega)=W_{G G M}(r, \Omega)-U_{G R S}(r, \Omega)= \\
& \frac{G M_{G G M}-G M_{G R S}}{r}+\frac{G M_{G G M}}{r} \sum_{n=2}^{n_{\max }}\left(\frac{a_{G G M}}{r}\right)^{n} \times \\
& \sum_{m=0}^{n}\left\{\Delta \bar{C}_{n m} \cos m \lambda+\bar{S}_{n m}^{G G M} \sin m \lambda\right\} \bar{P}_{n m}(\cos \theta),
\end{aligned}
$$

where the (residual) zonal harmonics are computed by

$$
\Delta \bar{C}_{n 0}=\bar{C}_{n 0}^{G G M}-\frac{G M_{G R S}}{G M_{G G M}}\left(\frac{a_{G R S}}{a_{G G M}}\right)^{n} \bar{C}_{n 0}^{G R S} .
$$

And the first term on the right-hand side of Eq. is the zero-degree scaling term of the disturbing potential. Therefore, the GGM-derived quantities need to account for the zero-degree term as well. For instance, the geoidal heights $N$ can be computed by inserting the disturbing potential (referred to the geoid level, with a geocentric radius $r_{g}$ ) into the well-known Bruns's formula: $N(\Omega)=T\left(r_{g}, \Omega\right)$ / $\gamma_{0}$, where $\gamma_{0}$ is the normal gravity on the reference ellipsoid. The zero degree geoid scaling term thus becomes:

$$
N_{0}(\Omega)=\frac{G M_{G G M}-G M_{G R S}}{r_{g} \gamma_{0}} .
$$

Recall, that the physical constants of the GRS-80 (Geodetic Reference System; Moritz 1992) are used for computing the normal gravity field parameters in the Baltic countries. Furthermore, the GPS-derived geodetic heights (an essential part of the GPS-levelling data!) are reckoned from the ETRS89 (new European Terrestrial Reference System) oriented GRS- 80 ellipsoid. The main parameters of the GRS-80 and the two tested geopotential models (EIGEN-GL04c and EGM96) are compared in Table 1.

Inserting $R=6371 \mathrm{~km}$ and $\gamma_{0}=981 \mathrm{Gal}$ into Eq. the zero degree geoid scaling term, associated with the EGM96 and EIGEN-GL04c, becomes $-0.936 \mathrm{~m}$, see Table 1 . This value will be added to the GGM-derived geoidal heights. Indeed, the geopotential models are particularly useful for computing the geoidal heights. As a matter of fact, a GGM can be considered as a realisation of the "world height system", which creates tools for unification of the national vertical datums all over the globe. As it will be shown later, the zero degree scaling term is detrimental in defining the offsets between the national vertical datums and the global gravimetric geoid.

Considering Bruns's formula and Eq. (3) the GGMderived height anomalies $\zeta$ (at the topographic surface, with the geocentric radius of $r_{t}=r_{g}+H$ ) will be computed thus by the following formula:

$$
\begin{aligned}
& \zeta_{G G M}\left(r_{t}, \Omega\right)=\frac{G M_{G G M}-G M_{G R S}}{r_{t} \gamma_{T}}+ \\
& \frac{G M_{G G M}}{r_{t} \gamma_{T}} \sum_{n=2}^{n_{\max }}\left(\frac{a_{G G M}}{r_{t}}\right)^{n} \times \\
& \sum_{m=0}^{n}\left\{\Delta \bar{C}_{n m} \cos m \lambda+\bar{S}_{n m}^{G G M} \sin m \lambda\right\} \bar{P}_{n m}(\cos \theta),
\end{aligned}
$$

where the normal gravity $\gamma_{T}$ is referred to the surface of the telluroid (with the geocentric radius of $r_{T}=r_{G R S}+$ $H$ ). Apparently, the higher $n_{\max }$ is applied in Eq. (6), the finer elements of the height anomalies can be sensed. As a matter of fact, the cumulative sum of a first few harmonic degrees in Eq. (6) contribute the most to the final (quasi)geoid value. For instance, the 10 first degrees provide some $70 \%$ of the final geoidal heights in the entire Nordic-Baltic region (Ellman 2004).

The conceptual differences between the geoid and heigh anomalies are well known, see e.g., Heiskanen, Moritz 1967: Chap. 8-3). In the Baltic countries the normal heights, thus related to the quasi-geoid, are used in surveying practice. The geoid to quasi-geoid separation is a function of Bouguer anomalies and the height (Heiskanen, Moritz 1967: 327). Recall, however, that over the Baltic countries these differences are numerically insignificant (a few mm only). This allows to neglect the geoid and quasigeoid differences in the following comparisons without affecting the objectives of the present study. In other words, we adopt $N(\Omega)=\zeta\left(r_{t}, \Omega\right)$ in the comparisons to follow.

\section{Global geopotential models}

\subsection{General}

A GGM comprises a set of fully-normalized, unitless spherical-harmonic coefficients $\left(\bar{C}_{n m}, \bar{S}_{n m}\right)$, which are obtained from the spectral analysis of the geopotential. For details of development and analysis of geopotential models see (Rapp, Pavlis 1990). In the past 40 years, many geopotential models have been estimated, for an extensive description of existing models we refer to (Bauman 2000) and the references therein.

In the compilation of a GGM the long-wavelength contribution of the Earth's gravity field is recovered from the satellite tracking data. Recall that the motion of a satellite is perturbed by various forces. By studying the satellite orbit perturbations the broad characteristics of the gravity field can be recovered. Improvements to the Earth gravity models at medium and short wavelengths should come from the use of satellite altimetry, terrestrial, marine or airborne gravity surveys - of varying

Table 1. The defining constants of the GRS-80, EGM96 and EIGEN-GL04c

\begin{tabular}{l|c|c|c|c}
\hline \multicolumn{1}{c|}{ Parameter } & Unit & EGM96 & EIGEN-GL04c & GRS-80 \\
\hline Equatorial radius, $a$ & $\mathrm{~m}$ & 6378136.3 & 6378136.46 & 6378137 \\
\hline Gravity Mass constant, $G M$ & $\mathrm{~km}^{3} \mathrm{~s}^{-2}$ & 398600.4415 & 398600.4415 & 398600.5 \\
\hline The zero degree geoid term, $N_{0}(\Omega)$ & $\mathrm{m}$ & -0.936 & -0.936 & $\mathrm{n} / \mathrm{a}$ \\
\hline
\end{tabular}


epoch, quality and geographic coverage. The accuracy of such models, at higher degrees is quite dependent on the geographic coverage of gravity data that goes into the solution. As the coverage improves, so will the model. Needless to mention that the weighting of the various data types in the GGM development is a delicate task. Prior to the year of 2000 the harmonic degree errors of the GGM-s were roughly divided into three frequency bands, cf. e.g. (Vaniček, Featherstone 1998):

1) Spherical harmonic degrees $2 \leq n \leq 20$ offer a superior information source of the low frequency component of the geoid. The estimation of the coefficients of these degrees is exclusively based on the satellite-derived contribution.

2) Spherical harmonic degrees $20<n \leq 120$, for which the GGM gives a reasonable accuracy almost everywhere on Earth and where the terrestrial data may offer an improvement in certain parts of the world, only if these data are of good quality.

3) Spherical harmonic degrees $120<n \leq 360$, for which the geopotential model may not be the best source of gravity field information and an improvement from terrestrial data should thus be sought. The degradation of the GGM in this region can be seen from the error degree variances, which are usually almost of the same magnitude as the gravity signal.

In the years 2000 and 2002, two dedicated satellite gravimetric missions started to operate. These missions are: CHAllenging Minisatellite Payload (CHAMP) for geoscientific and atmospheric research, managed by the Potsdam GFZ; and Gravity Recovery and Climate Experiment (GRACE), initiated by the National Aeronautics and Space Administration (NASA). The main goal of those missions is to provide the high-accuracy static and time variable (e.g., due to ocean currents and seasonal mass re-distribution) gravity fields. In this respect, the GRACE twin-satellites are outstandingly useful, which move on almost polar orbits and measure also the intersatellite range changes. The CHAMP and GRACE data have also been used for compilating the recent GGM-s. It is expected that the accuracy of the low and intermediate frequency bands of such models could be considered almost errorless compared to the terrestrial data errors. Section 3.2 and 3.3 contain a brief description of the EGM96 and EIGEN-GL04c geopotential models, which will be used in the numerical investigations.

\subsection{The Earth Geopotential Model EGM96}

It is commonly regarded, that the global accuracy of the EGM96 (Lemoine et al. 1998) was the best among other models of the past decade. The EGM96 harmonic coefficients are complete to degree and order 360 , which corresponds to the spatial resolution of $55 \mathrm{~km}$. The EGM96 derived geoidal heights are claimed to be accurate to 1 metre worldwide. The EGM96 is obtained from combining satellite tracking data, surface gravity data, and satellite altimeter measurements. The long-wavelength part of the Earth's gravity field was determined from various tracking measurements of orbiting satellites. The spatial resolution of the "satellite-only" solution is limited to about $600-700 \mathrm{~km}$, implying the highest harmoni- cs degree as of 35. The data in the EGM96 solution are of considerably varying vintage and quality, and of incomplete geographical coverage. Nevertheless, the EGM96 model represents a significant improvement over earlier models due to the release of new gravity data from formerly classified sources, as well as improved satellite tracking data.

\subsection{The GRACE Gravity Model EIGEN-GL04c}

GRACE tracking data is the basis of the new geopotential model EIGEN-GL04c (http://www.gfz-potsdam.de/ pb1/op/grace/results/grav/g005_eigen-gl04c.html), released by the Potsdam GFZ in March 31 2006. The model is a combination of the GRACE and LAGEOS space missions plus $0.5^{\circ} \times 0.5^{\circ}$ terrestrial gravimetry and altimetry surface data. This model (Förste et al. 2006) employs over 2 years of the GRACE tracking data gathered during $\mathrm{Fe}$ bruary 2003 through July 2005. The EIGEN-GL04C field is developed to degree and order 360. A special band-limited combination method has been applied in order to preserve the high accuracy from the satellite data in the lower frequency band of the geopotential and to form a smooth transition to the high frequency information coming from the surface data.

Due to the global, homogeneous nature of GRACE data, the resulting geoid errors show no discrimination between land and sea areas, as previous gravity models do. The EIGEN-GL04c geoid is estimated (Förste et al. 2006) to be accurate to approximately $3 \mathrm{~cm}$ to degree and order 100 (200 km resolution) and $30 \mathrm{~cm}$ to degree and order 360 ( $55 \mathrm{~km}$ resolution).

It should be noted, that the Centre for Space Research at the University of Texas has also developed a family of GRACE based GGM-s. However, these models are either with poorer resolution or use shorter GRACE data-spans.

\section{The study area and existing geoid models in the region of interest}

\subsection{Study area}

The location of the target area of this study is shown in Fig. 1. The performance of the two GGM-s is examined within the following geographical boundaries: $53.83^{\circ}<$ $\varphi<60.06^{\circ} ; 19.97^{\circ}<\lambda<28.52^{\circ}$. Thus, in addition to Estonia, Latvia and Lithuania, the target area includes partly also Russia, Belarus, Poland and Finland, together with a large portion of the Baltic Sea. The elevation extremes are $0 \mathrm{~m}$ at a shoreline and $318 \mathrm{~m}$ in southeast Estonia, whereas most of the target area comprises of sea and topography below $100 \mathrm{~m}$. Therefore, no significant numerical differences ( $3 \mathrm{~mm}$ at most) between the geoid and quasigeoid models exists over the chosen target area.

The extremes of the geoidal heights in the target area are 30 and $15 \mathrm{~m}$. They are located in the southwest and northeast corners, respectively (the length of this diagonal is $\sim 800 \mathrm{~km}$ ), thus the geoidal heights decrease from SW toward NE. The Baltic geoid model is mainly smooth (with a standard deviation (STD) of the mean $\sim 3 \mathrm{~m}$ ), but it includes some local irregularities in the NW part of the 


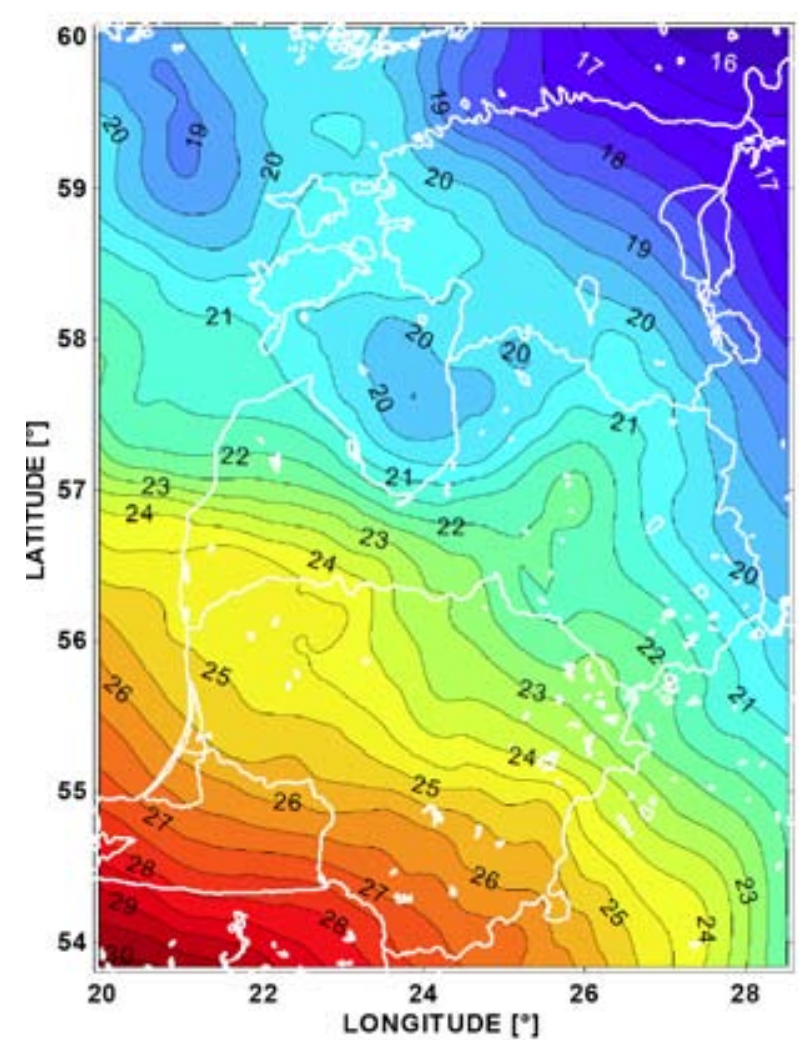

Fig. 1. The $1.5^{\prime} \times 3^{\prime}$ Baltic gravimetric geoid model BALTgeoid-04 (Ellmann 2004). Geoidal heights are given with respect to the GRS-80. Unit is metre and the contour interval is $0.5 \mathrm{~m}$. The total area of the image corresponds to $300000 \mathrm{~km}^{2}$

target area. Their location is correlated with the local anomalies of the gravity field (Ellmann 2004: Fig. 2.3).

\subsection{Pre-GRACE geoid models in the region of interest}

It is appropriate to review earlier geoid models for the same region. During the last two decades the geoid determination for the whole Nordic region has been carried out within the framework of the Nordic Geodetic Commission (NKG). Several NKG geoid models were delivered, see e.g. reference list in (Forsberg 2001). In 1990-ies the NKG geoid models were extended to the Baltic countries. Access to new gravity data from formerly classified sources, and release of EGM96, resulted in achieving better than a dm-accuracy for the regional NKG96 geoid model (Forsberg et al. 1997). Including the most recent terrestrial data, a new geoid model (Forsberg 2001)was computed using exactly the same computational setup as for the NKG96 model. The national geoid solutions, either for Estonia, Latvia or Lithuania, were published (Vermeer 1994; Kaminskis, Forsberg 1997; Forsberg 1998; Jürgenson 2001, 2003; Ellmann 2001). The study area is also covered by the European quasigeoid model EGG97 (Denker, Torge 1998). With a few exceptions (e.g. Vermeer 1994; Ellmann 2001; Denker, Torge 1998) the geoid computations were carried out by the NKG methodology (for a review see e.g. Forsberg 2001) and software. In particular, the full expansion $\left(n_{\max }=360\right)$ of the EGM96 model in conjunction with a very large integration domain (where available) was often utilised in the regional geoid modelling. It is believed that a good quality terrestrial data-set may somewhat compensate the shortages of the past geopotential models. However, Vaniček, Featherstone (1998) argue that this common assumption is not entirely flawless.

\subsection{Regional high-resolution BALTgeoid-04 model}

A Baltic geoid model was computed (Ellmann 2004; 2005) recently. The BALTgeoid-04 model was estimated by the least squares modified Stokes's formula (cf., Sjöberg 1991), whereas an early GRACE ("satellite-only") GGM01s model (Tapley et al. 2004) was employed as the reference. The quality of this gravimetric model was assessed from the comparisons with the GPS and levelling datasets. The same control points will be also used for the evaluation of the EGM96 and EIGEN-GL04c geoid models, therefore some more information on these data is spared for Section 5.2. The GPS-levelling points form a surface, which is called here the "geometric geoid model" $\left(N^{g e o m}=h-H\right)$. Four transformation parameters between the BALTgeoid-04 and the geometric geoid models were defined and thereafter a polynomial fit (Ellmann 2004: Eq. 23) was applied. The following STD-s of the post-fit residuals were achieved: Baltic $5.3 \mathrm{~cm}$, Estonia $2.8 \mathrm{~cm}$, Latvia $5.2 \mathrm{~cm}$ and Lithuania $4.3 \mathrm{~cm}$, respectively (see also the uppermost part of Table 3 ). It is also concluded that the accuracy of the BALTgeoid-04 model is at least of the same level as is the accuracy of the used control points (Ellmann 2005). In some of the following comparisons (see Tables 2 and 3) the BALTgeoid-04 will act in the capacity of the ground "truth".

\section{Numerical investigations}

\subsection{Comparisons of different spectral windows of the GGM-derived geoid models}

Several grids of the geoidal heights were computed over the target area by Eq. (6). Each of those grids utilises either different geopotential model (EIGEN-GL04c or EGM96) or degrees of expansion $n_{\max }$. Two $n_{\max }$ values are chosen for numerical investigations: $n_{\max }=95$ and $n_{\text {max }}=360$. This selection is not random; it is commonly appreciated that the degree $n=95$ is the uppermost reliable degree of the "GRACE-only" gravity field information. The limit $n_{\max }=360$ is the maximum degree of expansion of both combined GGM-s to be studied.

A spectral windows of the geoidal signal $N_{\mathrm{i} ; \mathrm{j}}^{\mathrm{GGM}}$ is obtained by subtracting the low-resolution contribution (extended up to $n_{\max }=\mathrm{i}-1$ ) from the one adopting an upper limit $n=\mathrm{j}$. In other words, we apply either lowpass or high-pass filtering approaches. The discrepancies $\Delta N_{\mathrm{i} ; \mathrm{j}}$ among the EIGEN-GL04c and EGM96 models are computed as:

$$
\Delta N_{\mathrm{i} ; \mathrm{j}}=N_{\mathrm{i} ; \mathrm{j}}^{\mathrm{EIGEN}}-N_{\mathrm{i} ; \mathrm{j}}^{\mathrm{EGM} 96},
$$

where the superscripts are associated with the utilized geopotential models. Numerical statistics for selected spectral windows and their discrepancies are presented in Table 2. Column 2 represents the BALTgeoid-04 related "true" values. 
Table 2. Numerical statistics of the selected spectral windows $\left(N_{\mathrm{i} ; \mathrm{j}}^{\mathrm{EIGEN}}\right)$ of the EIGEN-GL04c geopotential model and their discrepancies with the EGM96. Units are in metres

\begin{tabular}{|c|c|c|c|c|}
\hline \multirow{2}{*}{ Statistics } & \multirow{2}{*}{$\begin{array}{l}\text { BALTgeoid-04 } \\
\text { (Ellmann 2005), } \\
\text { without the zero-degree } \\
\text { scaling term }\end{array}$} & \multicolumn{3}{|c|}{ Contribution of $N_{\mathrm{i} ; \mathrm{j}}^{\mathrm{EIGEN}} /$ Discrepancies: $\Delta N_{\mathrm{i} ; \mathrm{j}}=N_{\mathrm{i} ; \mathrm{j}}^{\mathrm{EIGEN}}-N_{\mathrm{i} ; \mathrm{j}}^{\mathrm{EGM} 96}$} \\
\hline & & $N_{2 ; 360}^{\mathrm{EIGEN}} / \Delta \mathrm{N}_{2 ; 360}$ & $N_{2 ; 95}^{\mathrm{EIGEN}} / \Delta \mathrm{N}_{2 ; 95}$ & 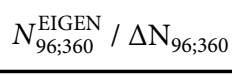 \\
\hline Maximum & 30.17 & $+30.05 /+0.91$ & $+29.57 /+0.56$ & $1.37 /+0.53$ \\
\hline Minimum & 15.60 & $+15.14 /-0.62$ & $+15.73 /-0.14$ & $-1.90 /-0.52$ \\
\hline Mean & 22.04 & $+22.07 /+0.23$ & $+22.02 /+0.21$ & $+0.05 /+0.02$ \\
\hline Standard deviation of the mean & 2.97 & $2.98 / 0.23$ & $2.93 / 0.17$ & $0.56 / 0.15$ \\
\hline
\end{tabular}

The results in Table 2 need to be discussed more explicitly. As is expected, the shape (not shown here) and magnitude of the long wavelength (i.e. "satellite-only") contribution of EIGEN-GL04c derived geoidal heights, $N_{2 ; 95}^{\text {EIGEN }}$, are rather similar (cf. columns 2 and 4, Table 2) to the "true" geoid (here represented by the BALTgeoid-04), cf. Fig 1. Roughly, the first 95 degrees provide almost $90 \%$ from the final geoid model over the Baltic countries. By inclusion the contribution of the harmonic degrees $96 \leq n \leq 360$, the range of the geoidal heights change, but little. Note that the magnitude of the spectral window of $N_{96 ; 360}^{\mathrm{EIGEN}}$ varies from -1.9 to $+1.4 \mathrm{~m}$ only (cf. Table 2, column 5). However, in a regional average the contribution of $N_{96 ; 360}^{\text {EIGEN }}$ is almost a zero.

The discrepancies between the EIGEN-GL04c and EGM96 geoid grids at harmonic degree 95, $\Delta N_{2 ; 95}$, are portrayed in Fig. 2. The amplitude of $\Delta N_{2 ; 95}$ varies several decimetres, whereas the largest (positive) discrepancies $(>0.5 \mathrm{~m})$ are located in West Latvia and over the Gulf of Riga. Importantly, over the entire study area the mean of the discrepancies exceeds $+0.2 \mathrm{~m}$ (see Table 2 , column 4 ). This mean value is also associated with $\Delta N_{2 ; 360}$ (see Table 2 , column 3). Obviously, the long wavelength $\Delta N_{2 ; 95}$ contributes the most to the $\Delta N_{2 ; 360}$ magnitude.

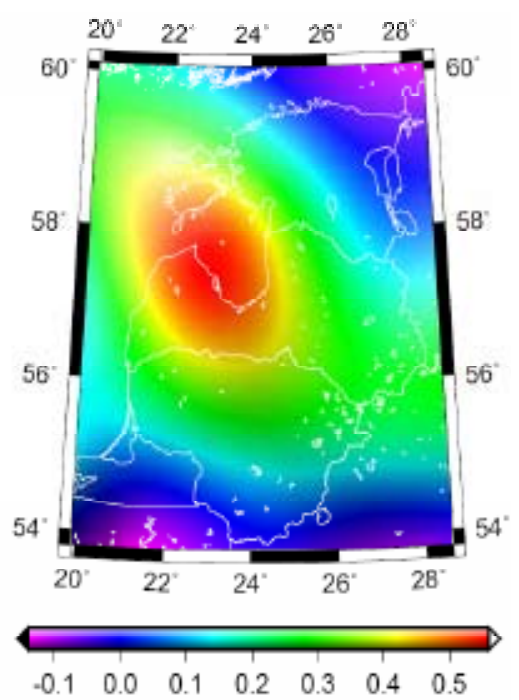

Fig. 2. Discrepancies between the spectral window 2-95 of the EIGEN-GL04c and EGM96 geoid models, $\Delta N_{2 ; 95}$. The discrepancies range from $-0.14 \mathrm{~m}$ to $+0.56 \mathrm{~m}$ with a mean of $+0.21 \mathrm{~m}$. Generally, the EIGEN-GL04c geoid appears to be higher than that of the EGM96 model. Standard deviation of the detected discrepancies amounts to $0.17 \mathrm{~m}$
This is supported by the fact that for the high-pass spectral window (i.e., the $N_{96 ; 360}^{\text {EIGEN }}$ contribution) the mean of discrepancies is almost a zero.

Since the spectral window $N_{2: 95}^{\mathrm{EGM} 96}$ of the EGM96 model contains historical and insufficiently covered (especially in sub-polar latitudes) satellite tracking data, then most likely the detected discrepancies are due to the GRACE-induced enhancements.

The discrepancies $\Delta N_{96 ; 360}$ vary within \pm 0.5 (see column 5, Table 2). It should be noted that the geographical distribution and appearance of the $\Delta N_{96 ; 360}$ highs and lows (not shown here) are rather random, with a regional average as of $+0.02 \mathrm{~m}$ (cf. column 5 , Table 2 ). The most significant discrepancies are located over offshore, though. This may indicate the presence of the systematical biases among the datasets used for the compilation of the EIGEN-GL04c and EGM96 models.

Indeed, note that the NKG 1997 marine and Baltic Sea 1999 aero-gravity surveys (Forsberg 2001) have significantly improved the "post-EGM96" data coverage over the Baltic Sea. In addition, the marine gravity data within the Riga Gulf and nearby Latvian coastline have been made available as well. For the geographical distribution of the up-to-date gravity datapoints see (Ellmann 2005: Fig. 2). Conversely, at the compilation of the EGM96 the only source available over the Baltic Sea was the satellite altimetry results. Evidently, the recent improvements in the data coverage have been considered by the EIGENGL04c solution. In the mainland the absolute range of the $\Delta N_{96 ; 360}$ discrepancies (between the EIGEN-GL04c and EGM96 models) remains within $\pm 2 \mathrm{dm}$. In other words, over such areas more or less similar datasets were employed at the compilation of both GGM-s.

The final $\Delta N_{2 ; 360}$ discrepancies between the EIGENGL04c and EGM96 geoid models (cf. Eq. (6)) are depicted in Fig. 3. The complete statistics of the comparison can be found in Table 2. Here we focus only on the general features of the discrepancies between the corresponding geoidal heights. The range of the detected discrepancies varies from $-0.6 \mathrm{~m}$ up to $+0.9 \mathrm{~m}$. The largest discrepancies are located in West-Latvia and over the Baltic Sea. Obviously, the main contributors to this are: $(i)$ the long wavelength improvements due to the GRACE satellites, (ii) the higher $(>90)$ wavelengths have been affected by the new data over the Baltic Sea.

Summarizing this section, the features of combined models, such as EGM96 and EIGEN-GL04c, are quite dependent on geographical coverage of gravity data that go into the solution. The range of discrepancies between the EIGEN-GL04c and EGM96 models may reach up to 


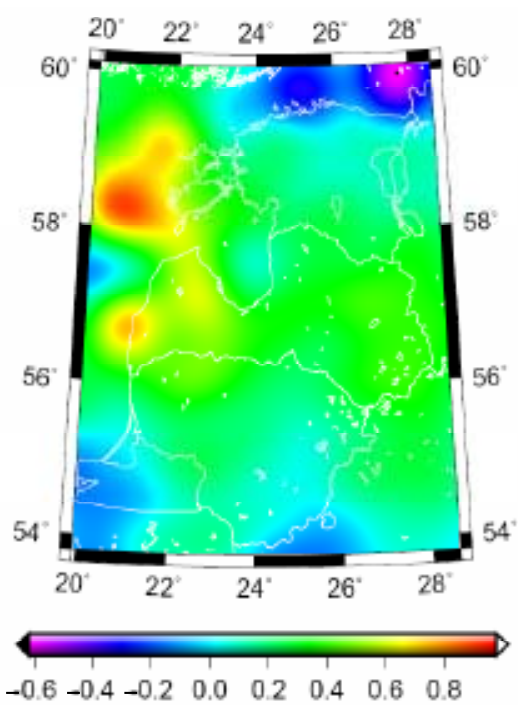

Fig. 3. Discrepancies between the whole spectra of the EIGEN-GL04c and EGM96 geoid models, $\Delta \mathrm{N}_{2 ; 360}$. The discrepancies range from $-0.62 \mathrm{~m}$ to $+0.91 \mathrm{~m}$ with a mean of $+0.23 \mathrm{~m}$. Generally, the EIGEN-GL04c geoid appears to be higher than that of the EGM96 model. Standard deviation of the detected discrepancies amounts to $0.23 \mathrm{~m}$

a meter in the study area. However, different geoid modelling results can be expected in different regions. One may wonder, which model under the study is best suited for the region of interest, or, in other words, which one is better corresponding to the ground "truth". For assessment of geoid models the comparison with the GPS and levelling datasets is very useful. The results of relevant tests are presented in the next section.

\subsection{Comparisons with the GPS-levelling data}

As is well known, inter-comparison of a geoid model, GPS-derived geodetic heights, and spirit-levelled (normal or orthometric) heights at discrete points gives a reasonable indication of the geoid model's accuracy. Thus the further validation of the two GGM models relays on nationwide sets (one for each country) of high-precision geodetic points, for their locations see Fig. 4. It should be noted that the same constellation of the control points as used at the evaluation of the BALTgeod-04 model will also be employed here. For all points the geodetic heights from GPSmeasurements as well as levelling heights are available. The geodetic coordinates of the control points are related to the respective national realization of the new European Terrestrial Reference System ETRS-89. The spirit-levelled normal heights are given in the Baltic Height System 1977, which refers to the Kronstadt tide-gauge (located in the vicinity of St. Petersburg, $\varphi \approx 60^{\circ} \mathrm{N}$ and $\lambda \approx 30^{\circ} \mathrm{E}$ ).

The average distance between 26 evenly distributed Estonian control points is $50 \mathrm{~km}$. The combined error of GPS-derived and spirit-levelled heights does not exceed $2-3 \mathrm{~cm}$, most likely. Note that the geodetic heights are computed from the same GPS campaign and most of these points are directly connected to the high-precision levelling network. The Latvian and Lithuanian datasets (53 and 110 points, respectively) are denser. However, due to inclusion of lower-order control networks, the

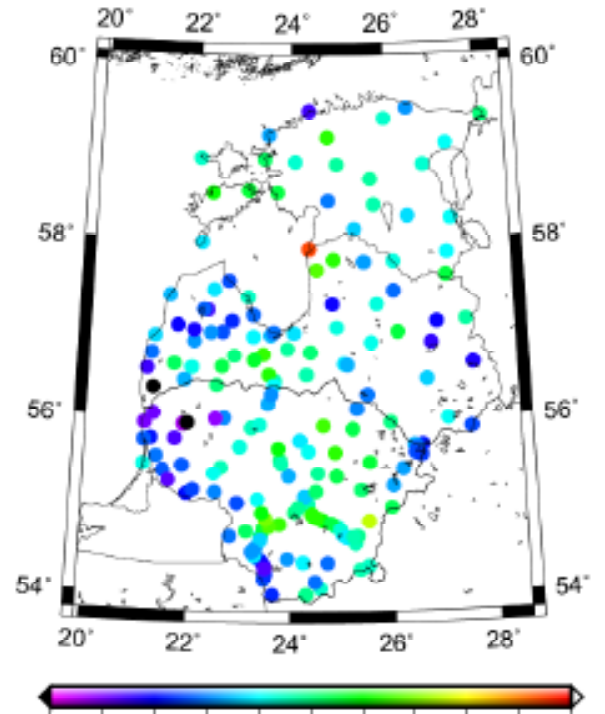

$\begin{array}{llllllllllll}0.1 & 0.2 & 0.3 & 0.4 & 0.5 & 0.6 & 0.7 & 0.8 & 0.9 & 1.0 & 1.1\end{array}$

Fig. 4. Distribution of the Baltic GPS-levelling data (altogether 189points) and their differences from the EIGEN-GL04c geoidal heights (developed to $n=360$ ). The discrepancies $\left(N^{\text {geom }}-N_{2 ; 360}^{\mathrm{EIGEN}}\right)$ range from +0.04 to $+1.04 \mathrm{~m}$, with a mean of $+0.53 \mathrm{~m}$. The standard deviation of the discrepancies amounts to $0.157 \mathrm{~m}$. The colours of the data-points are proportional to the range of the detected discrepancies (cf. the colourbar). Unit is metre. The levellings are referred to the Kronstadt tide-gauge

accuracy of the used GPS-levelling points seems to be rather heterogeneous there.

The common Baltic geometric geoid is represented by the sum of the three national datasets (189 points). Both GGM-s are developed up to $n_{\max }=360$ (resolution $55 \mathrm{~km}$ ) to calculate the gravimetric geoidal heights $N^{\text {grav }}$ by Eq. (6) at the locations of the control points. The numerical statistics of the detected differences among the GGM-derived and geometrical geoid models are presented in Table 3. In particular, the mean of the differences reveals positive offsets of the Kronstadt vertical datum from the GGM-derived global geoid (+0.49 and $+0.73 \mathrm{~m}$ for the EIGEN-GL04c and EGM96, respectively). The resulting STD of differences ( 0.15 and $0.20 \mathrm{~m}$ for the EIGEN-GL04c and EGM96, respectively) show that the achieved accuracy is almost three - four times worse than that of the regional BALTgeoid-04 (Ellmann 2005). The magnitude of exposed differences between geometric and EIGEN-GL04c-derived geoid model, $\delta N=$ $N^{\text {geom }}-N_{2: 360}^{\text {EIGEN }}$, range from +0.04 to $+1.04 \mathrm{~m}$, see Fig. 4 and Table 3. The EGM96 associated discrepancies (not shown here) are more scattered, see Table 3.

The comparisons of the GGM-s and the national GPS-levelling data may be affected by systematic effects (e.g., due to land uplift) and datum inconsistencies. Now we attempt to minimize the offsets (i.e. vertical offset and possible tilt) between the gravimetric and geometrical geoid models by introducing a four-parameter polynomial fit, cf. (Forsberg 2001). The GPS-levelling points are used for definition of the transformation parameters among the geometrical and the GGM-derived geoid models. Thereafter, these parameters were applied for fitting the GGMderived geoidal heights with the GPS-levelling points. The 
Table 3. Numerical statistics of comparisons between the geoid models and GPS-levelling points. Units are in metres

\begin{tabular}{|c|c|c|c|c|c|c|c|c|}
\hline \multirow{2}{*}{ MODEL } & \multirow{2}{*}{ COUNTRY } & \multicolumn{4}{|c|}{ Difference $\delta N=N^{\text {geom }}-N^{\text {grav }}$} & \multicolumn{3}{|c|}{ Post-fit residuals } \\
\hline & & Min & $\operatorname{Max}$ & Mean & STD & Min & Max & STD \\
\hline \multicolumn{9}{|c|}{ GPS-levelling (Kronstadt vertical datum) } \\
\hline \multirow{4}{*}{ BALTgeoid-04 $\left(1.5^{\prime} \times 3^{\prime}\right)$} & Baltic (189 points) & 0.39 & 0.70 & +0.532 & 0.058 & -0.13 & 0.16 & 0.053 \\
\hline & Estonia (26 points) & 0.45 & 0.62 & +0.553 & 0.040 & -0.06 & 0.04 & 0.028 \\
\hline & Latvia (53 points) & 0.42 & 0.70 & +0.542 & 0.060 & -0.10 & 0.14 & 0.054 \\
\hline & Lithuania (110 points) & 0.39 & 0.65 & +0.514 & 0.057 & -0.09 & 0.14 & 0.046 \\
\hline \multirow{4}{*}{ EIGEN-GL04c $\left(n_{\max }=360\right)$} & Baltic (189 points) & 0.04 & 1.04 & +0.487 & 0.157 & -0.37 & 0.53 & 0.148 \\
\hline & Estonia (26 points) & 0.24 & 1.04 & +0.548 & 0.144 & -0.25 & 0.42 & 0.135 \\
\hline & Latvia (53 points) & 0.04 & 0.76 & +0.451 & 0.146 & -0.27 & 0.27 & 0.130 \\
\hline & Lithuania (110 points) & 0.05 & 0.84 & +0.474 & 0.161 & -0.27 & 0.33 & 0.118 \\
\hline \multirow{4}{*}{ EGM96 $\left(n_{\max }=360\right)$} & Baltic (189 points) & 0.20 & 1.23 & +0.728 & 0.205 & -0.45 & 0.48 & 0.151 \\
\hline & Estonia (26 points) & 0.20 & 1.23 & +0.757 & 0.254 & -0.39 & 0.26 & 0.145 \\
\hline & Latvia (53 points) & 0.56 & 1.20 & +0.894 & 0.162 & -0.40 & 0.26 & 0.140 \\
\hline & Lithuania (110 points) & 0.34 & 1.09 & +0.640 & 0.154 & -0.20 & 0.38 & 0.107 \\
\hline \multicolumn{9}{|c|}{ GPS-levelling (Amsterdam vertical datum) } \\
\hline EIGEN-GL04c $\left(n_{\max }=360\right)$ & Lithuania (223 points) & -0.01 & 0.68 & +0.376 & 0.123 & -0.19 & 0.29 & 0.098 \\
\hline EGM96 $\left(n_{\max }=360\right)$ & Lithuania (223 points) & 0.27 & 1.09 & +0.598 & 0.146 & -0.20 & 0.43 & 0.119 \\
\hline
\end{tabular}

A geometric geoid height, $N^{\text {geom }}$, is obtained by algebraically subtracting the levelling height from a GPS derived height. The geoidal heights $N^{\text {grav }}$ at the GPS-levelling points are computed from the EGM96 and EIGEN-GL04c geopotential models at their uppermost limit $\left(n_{\max }=360\right.$ ), or by interpolation from the closest grid points of the high-resolution BALTgeoid-04 model. Note that the mean of the post-fit residuals is a zero for all the cases.

numerical statistics of this fitting are presented on the right hand side of Table 3 . In particular, the range of postfit residuals for the EIGEN-GL04c geoid varies from -0.37 to $+0.53 \mathrm{~m}$ (cf. Table 3). The achieved STD of the post-fit residuals is $\sim 0.15 \mathrm{~m}$ for both GGM-s under study.

Note that the STD of the post-fit residuals is reduced, of course, compared to the corresponding STD value of (pre-fit) differences $\delta N$ (cf. Table 3). Intuitively, the less the difference between pre- and post-fit statistics, the better the GGM geoid model agrees with the practical realization of the vertical datum. An insignificant difference of the STD and post-fit residuals indicate most likely an one-dimensional offset between the GPS-levelling data and a geoid model. Such parallelism was detected between the practical realisation of the national vertical datum and the EIGEN-GL04c geoid. Thus the EIGENGL04c gives the impression of greater reliability, in contrast to somewhat tilted, the EGM96 based geoid model. This comparison indicates that the EIGEN-GL04c is superior to the EGM96 in the target area. The further discussion thus focuses on the EIGEN- GL04c results.

Such comparisons are also produced on a countryby-country basis. The corresponding statistics can be found in Table 3. In particular, the STD of the EIGENGL04c post-fitting residuals as of $0.12 \ldots 0.13 \mathrm{~m}$ were achieved for the Estonian, Latvian and Lithuanian GPSlevelling points. Again the resulting STD of post-fit residuals shows that the achieved accuracy is almost threefour times worse than the accuracy of the BALTgeoid-04 regional model.

Summarizing, a decimetre level accuracy can be obtained for the EIGEN-GL04c-derived geoid model, provided that any datum inconsistencies have been eliminated. The results in Table 3 indicate that the resolution of the contemporary geopotential models is still not satisfactory for the height determination with the geodetic accuracy.
Note that the Estonian GPS-levelling geoid appears to be somewhat "higher" than the Latvian and Lithuanian geometrical geoid models, see the mean values $(+0.55 \mathrm{~m}$ versus $+0.45 \ldots+0.47 \mathrm{~m})$ in Table 3 . It should be noted that for simplicity no temporal changes in the levelled heights were considered in this study. The Estonian territory is affected by the Fennoscandian postglacial rebound. Conversely, the Latvian and Lithuanian points are located outside the land-uplift zone. Since the levellings have been performed over relatively long timespan then the Estonian solution may be contaminated with the land-uplift effect.

Thus, the GPS-levelling points cannot be considered as an entirely errorless dataset. The following exercise is a clear demonstration of this. Recently, an updated set of the GPS-levelling data (altogether 223 points) became available for Lithuania (pers.comm., E. Parseliunas, Aug. 2007), see Fig. 5. The comparison with the EIGEN-GL04c geoidal heights yields the significantly reduced STD of differences: $0.123 \mathrm{~m}$ (cf. with $0.161 \mathrm{~m}$, which was achieved from the comparison with the "old" set of control points, see Table 3). Note also the different values of the achieved mean. The new set of the control points shows that the offset from the EIGEN-derived global geoid has now been reduced to $+0.376 \mathrm{~m}$ (cf. with $+0.474 \mathrm{~m}$, which was achieved from the comparison with the "old" set of control points, see Table 3). This is due to the fact that the levelled normal heights of the "new" control points are referred to the sea level observations at the Amsterdam tide-gauge (located at $\varphi \approx$ $52.5^{\circ} \mathrm{N} \& \lambda \approx 5^{\circ} \mathrm{E}$ ), which is also being used in the UELN (United European Levelling Network) adjustments. The detected difference of the mean values, $+10 \mathrm{~cm}$ is in good agreement with the directly levelled differences between the Kronstadt and Amsterdam vertical datums (see, e.g., Ihde et al. 2001). Even though the data at these tide- 
gauges are acquired during different time spans, this is a direct evidence that the mean sea level (due to sea surface topography) at the eastern shore of the Baltic Sea is higher than that of the North Sea. Consequently, the EIGEN-GL04c resolution and accuracy seem to be sufficient to provide an approximate estimate for the offsets among different vertical datums.

Intuitively, future high-resolution and more accurate global geoid models create tools for unification of national height systems all over the globe. One such a candidate is a new high-resolution Earth Gravitational Model, EGM07, which is currently under development (Pavlis et al. 2007). This new EGM07 will take advantage of updated satellite, terrestrial gravity, elevation and altimetry data, the project is sponsored by the National Geospatial-Intelligence Agency of the USA. The resolution of the EGM07 is 5' arc-minutes (corresponding to $9 \mathrm{~km}$, i.e. to the degree of 2160), as a result also the EGM07 accuracy is expected to be superior over earlier GGM-s. In addition to the geodetic applications, the EGM07 will also contribute to the studies of the Earth's interior, the long-term geophysical processes (post-glacial rebound, plate tectonics, mantle convection, etc.) and to oceanography.

\section{Summary and conclusions}

High-precision tracking data of the orbital perturbations of the GRACE twin-satellites provide accurate gravity information with spatial resolution of $200 \mathrm{~km}$. This data is on the basis of the new geopotential model EIGENGL04c, which was released in March 2006. The range and distribution of different spectral windows of the EIGENGL04c geoid was compared with that of the EGM96 model. The detected discrepancies between the whole spectra of the two models vary from $-0.6 \mathrm{~m}$ up to $+0.9 \mathrm{~m}$. The largest discrepancies are located in West-Latvia and over the Baltic Sea. Obviously, the main contributors to this are: (i) the long wavelength improvements due to the GRACE satellites, $(i i)$ the higher wavelengths have been affected by the new data over the Baltic Sea.

Further numerical tests involved several sets of high precision GPS-levelling points from Estonia, Latvia and Lithuania. There are some notable improvements of numerical statistics (assessed by the GPS-levelling data) in the target area when utilizing the new EIGEN-GL04c, instead of using the EGM96. A decimetre level accuracy can be obtained for the EIGEN-GL04c derived geoid model, provided that any datum inconsistencies have been eliminated. Unlikely to the EGM96 geoid, the more realistic one-dimensional offset between the EIGEN-GL04c geoid and the height system is identified in the target area.

Another conclusion is that the EIGEN-GL04c comparisons with two different GPS-levelling data-sets provided a realistic estimate for offsets between the Amsterdam and Kronstadt vertical datums. The results of this study seem to support common expectations that the offset between the national vertical datums can be defined from the comparisons with the geoid models derived from the new satellite data. Thus, the future global geopotential models could lead to unification of different height systems not only within a continent but also overseas. In this respect of particular interest is the first

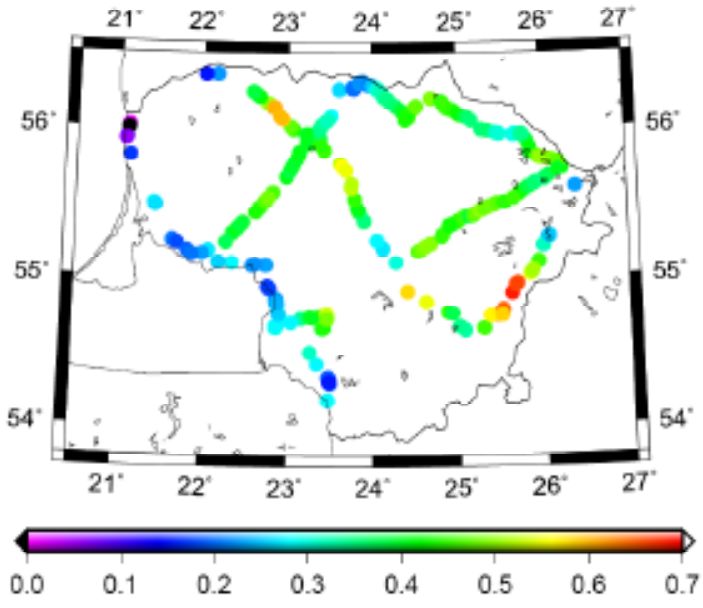

Fig. 5. Distribution of the "new" set of Lithuanian GPSlevelling data (altogether 223 points) and their differences from the EIGEN-GL04c geoidal heights (developed up to degree 360$)$. The discrepancies $\left(N_{\text {geom }}-N_{2 ; 360}^{\mathrm{EIGEN}}\right)$ range from -0.01 to $+0.68 \mathrm{~m}$, with a mean of $+0.376 \mathrm{~m}$. The STD of the discrepancies amounts to $0.123 \mathrm{~m}$. The colors of the data-points are proportional to the range of the detected discrepancies (cf. the colorbar). The unit is metre. The levellings are referred to the Amsterdam tide-gauge observations

satellite gradiometric mission GOCE (Gravity field and steady-state Ocean Circulation Explorer) to be launched by the European Space Agency in 2008. This mission will make available unprecedented accuracy for geopotential coefficients in the global scale and up to degree and order 270 (corresponding to the spatial resolution of $65 \mathrm{~km}$ ). The future GRACE models utilizing data from longer time span become also very useful.

An appropriate geopotential model is also essential to determine the regional gravimetric geoid model accurately. The present study results indicate that the EIGEN-GL04c based regional models may possess a great potential in providing further refinement of the regional geoid models.

\section{Acknowledgements}

The prime author is funded by the European Union Structural Funds, Project no. 1.0101-0335; the second author is supported by Estonian Science Foundation, grant no 5731. Dr. E. Parseliunas from the Vilnius Technical University, Lithuania and MSc. J. Kamiskis from the Latvian Geospatial Information Agency, are acknowledged for providing the GPS-levelling data.

\section{References}

Bouman, J. 2000. Quality assessment of satellite-based global gravity field models. Netherlands Geodetic Commission, Publications on Geodesy 48, Delft.

Denker, H.; Torge, W. 1998. The European gravimetric quasigeoid EGG97 - an IAG supported continental enterprise, in Forsberg, R.; Feissel, M.; Dietrich, R. (eds). Geodesy on the Move. IAG Symp. Series 119, Springer Verlag, 249-254.

Ellmann, A. 2001. Least squares modification of Stokes formula with applications to the Estonian geoid. Royal Institute of Technology, Division of Geodesy, Report No. 1056, Stockholm. 
Ellmann, A. 2004. Effect of the GRACE satellite emission on gravity field studies in Fennoscandia and the Baltic Sea region, in Proceedings of the Estonian Academy of Science, Geology, GRACE study 53: 67-93.

Ellmann, A. 2004. The geoid for the Baltic countries determined by the least squares modification of Stokes' formula. Royal Institute of Technology, Department of Infrastructure, Geodesy Report No. 1061, Stockholm.

Ellmann, A. 2005. Two deterministic and three stochastic modifications of Stokes's formula: a case study for the Baltic countries, Journal of Geodesy 79: 11-23.

Forsberg, R. 1998. Geoid tailoring to GPS - with example of a 1-cm geoid of Denmark, in Vermeer, M.; Ádám, J. (eds). Second continental Workshop on the geoid in Europe. Budapest, 10-14 March, Reports of the Finnish Geodetic Institute 98(4): 191-197.

Forsberg, R. 2001. Development of a Nordic cm-geoid with basics of geoid determination, in Harsson B. G. (ed.). Nordic Geodesy towards the $21^{\text {st }}$ century. Lecture notes for Autumn School, Nordic Geodetic Commission, Statens kartverk, Geodetic Publication 2001(1): 67-88.

Forsberg, R.; Kaminskis, J.; Solheim, D. 1997. Geoid for the Nordic and Baltic Region from gravimetry and satellite altimetry, in Segawa, J.; Fujimoto, H.; Okubo, S. (eds.). Gravity, Geoid and Marine Geodesy. IAG Symp. Series 117, Springer Verlag, 540-547.

Förste, C. et al. 2006. A mean global gravity field model from the combination of satellite mission and altimetry/gravimetry surface data: EIGEN-GL04c, Geophysical Research Abstracts 8: 03462.

Heiskanen, W. A.; Moritz, H. 1967. Physical geodesy. W. H. Freeman and Company. San Francisco.

Ihde, J.; Adam, J.; Gurtner, W.; Harsson, B. G.; Sacher, M.; Schlüter, W.; Wöppelmann, G. 2001. The height solution of the European Vertical GPS reference network (EUVN), in Proceedings of the EUREF Symposium in Tromsø, June 2224, 2000. Veröff. Bayer. Kom. Int. Erdmess. Bayer. Akad. Wiss.: Astron.-geod. Arb., München, 116-125.

Jürgenson, H. 2001. Gravity measurements and calculations of the geoid model for Estonia, in Ingemann, V.; Madsen, F. (eds.). Proc from Seminar of the Danish \& Baltic sector Programme, Riga, 1999, Copenhagen, 94-110.

Jürgenson, H. 2003. Determination of Estonian precision geoid, $\mathrm{PhD}$ Thesis, Estonian Agricultural University, Tartu.

Kaminskis, J.; Forsberg, R. 1997. A new detailed geoid for Latvia, in Segawa, J.; Fujimoto, H.; Okubo, S. (eds.). Gravity, Geoid and Marine geodesy. IAG Symposium Series 117, Springer Verlag, 621-628.

Kirby, J. F.; Featherstone, W. E. 1997. A study of zero- and firstdegree terms in geopotential models over Australia, Geomatics Research Australasia 66: 93-108.

Lemoine, F. G. et al. 1998. The development of the joint NASA, GSFC and NIMA Geopotential Model EGM96. NASA/TP-
1998-206861, NASA Goddard Space Flight Center, Greenbelt Maryland, USA.

Molodenskii, M. S.; Eremeev, V. F.; Yurkina, M. I. 1960. Methods for study of the external gravitational field and figure of the Earth (translated from Russian 1962). Israel program for scientific translations, Jerusalem.

Moritz, H. 1992, Geodetic Reference System 1980, Bull Geod 66: 187-192.

Pavlis, N. K.; Holmes, S. A.; Kenyon, S. C.; Factor J. K. 2007. Earth gravitational model to degree 2160: Status and progress. Presented at the IUGG XXIV General Assembly, Perugia, Italy.

Rapp, R; Pavlis, N. K. 1990. The development and analysis of geopotential coefficient models to spherical harmonic degree 360, J Geophys R 95(B13), 21885-21911.

Sjöberg, L. E. 1991. Refined least-squares modification of Stokes formula, Manuscr Geod 16: 367-375.

Stokes, G. G. 1849. On the variation of gravity at the surface of the earth, Trans. Cambridge Philos. Soc., VIIII: 672-695.

Tapley, B. D.; Bettadpur, S.; Watkins, M. M.; Reigber, Ch. 2004. The Gravity recovery and climate experiment: mission overview and early results, Geophys. Res. Lett. 31, L09607, DOI:10.1029/ 2004GL019920.

Vaníček, P.; Kleusberg, A. 1987. The Canadian geoid, Stokesian Approach. Manuscr Geod 12: 86-98.

Vaníček, P.; Featherstone, W. E. 1998. Performance of three types of Stokes's kernel in the combined solution for the geoid, Journal of Geodesy 72: 684-697.

Vermeer, M. 1994. A fast delivery GPS-gravimetric geoid for Estonia. Reports of Finnish Geodetic Institute, No 94:1, Helsinki.

Artu ELLMANN. Assoc. Prof., Tallinn University of Technology, Dept of Transportation, Geodesy Chair. Ph. +372 6202 603, Fax +3726202601.

He holds (since 2004) a $\mathrm{PhD}$ degree from the Royal Institute of Technology (KTH) in Stockholm. 2004-2006, a research fellow at the Geodetic Research Laboratory, University of New Brunswick, Canada. Over 30 presentations at international conferences, author/co-author of over 60 publications in referred Journals and Conference Proceedings, technical reports, magazine articles.

Research interests: physical geodesy, gravity field and geoid modelling in particular, national geodetic networks.

Harli JÜRGENSON. Assoc. Prof., Estonian University of Life Sciences, Dept of Geomatics, Ph +3727313119 , Fax +372 313154.

PhD degree since 2003 from Estonian University of Life Sciences in Tartu. Ass. Prof. since 2003. Over 15 presentations in international conferences and seminars, author of over 20 publications in referred Conference Proceedings, technical reports, magazine articles.

Research interest: gravity field and geoid modelling, height datums. 